\title{
Blaschkoian Lichen Planus in an Amazghi; A Rare Case Report
}

\author{
Ebtisam Elghblawi*
}

Dermatology Department, St. James Hospital, Tripoli, P.O. Box 3232, Libya

\begin{abstract}
Lichen planus (LP) is a common acquired inflammatory dermatosis of unknown origin with several morphological forms. Linear lichen planus is frequently seen but cases of zonal/ zosteriform/ dermatomal/ blaschkoid LP are rare.

Typically LP is merely acknowledged as an old companion to a dermatologist in the clinic. Nonetheless sometimes it comes in masquerades. Many reports have documented LP to occur in scars of previous herpes zoster (HZ) lesions. Zosteriform LP without evidence of HZ is an extremely rare occurrence in the head and neck area. To the best of my knowledge such a condition has not been reported in the literature previously.

I report a healthy 53-year old Amazaghi woman with grouped lichenoid, unilateral linear plaque skin lesion on the right side of the face that followed the lines of Blaschko with no previous history of HZ on the involved area. The punch biopsy specimen confirmed the typical histology of lichen planus. A clinical diagnosis was made based on the clinical picture and biopsy reading.

Usually Blaschko linear skin lesions develop later in life with distinctive features, and thus it should be differentiated from other acquired skin dermatoses. I report this case of LP type for its scarcity and to add one more case to the list of existing literature, with the idiosyncrasy of late presentation onset.
\end{abstract}

Keywords: Blaschko's line, lichen planus, linear, unilateral.

\section{INTRODUCTION}

Lichen planus (LP) is a dermatosis of unknown origin with distinctive clinico-pathological attributes [1]. Unilateral LP follows Blaschko's lines (BL). The concept of BL has been suggested to be a congenital error of morphogenesis due to a single gene mutation or a stretched skin during the embryogenesis [2].

BL exist in an individual, and demonstrate a form of human 'mosaicism' in which there are two or more diverse cell populations, initiated from a sole zygote [3]. BL have explicit outlines on each side of the body.

The typical characteristic Lichen planus (LP) is an inflammatory muco-cutaneous disease where the cellmediated immunity (CMI) takes part in initiating and triggering the disease activity [4]. When LP is activated, most $\mathrm{T}$ cells will be heavily infiltrating in the lesion and around the lesion [4]. Moreover, typical LP is enormously itchy, with typical flat topped and polygonal violaceous colour plaques and papules [4].

\section{CASE PRESENTATION}

An Amazaghi 53-year-old female who is diabetic, presented with multiple eruptions of mildly pruritic violaceous lesions confined to the right side of the face for one month's duration. It started from the neck and then

*Address correspondence to this author at the Dermatology Department, St James Hospital, Tripoli, P.O. Box 3232, Libya; Tel: 4772531;

E-mail: ebtisamya@yahoo.com gradually progressed over the upper face and cheek area as the image shows (Figs. 1, 3, 4). Her past medical history was only significant for her diabetes.

There was no history of drug intake apart from the diabetic medication namely; Metformin $500 \mathrm{mg}$ three times a day and Amaryl 4mg which has active ingredients of 'Glimepiride' and is taken once before meals for her diabetes, it is often used in diabetes type 2, and Rosuvastatin $10 \mathrm{mg}$ once per day.

There was no history of trauma, or prior dermatological disease, and no history of any topical application. There was no family history of identical skin disease. She has been diabetic for the last four years. She did not have any other known co-morbidities.

On physical examination, the skin lesions were unilateral, multiple and discrete, localized to the right side of the face, covering the right apical part of the forehead near the hair line (1-3 mm), right temple, under right ear, and right cheek and on the right neck side as well. The lesion over the forehead was slightly raised, flat topped, a violaceous lichenoid thick plaque with scales overlying it without specific configuration. The lesion over the cheek was macular without specific configuration, and the right neck side was plaque, with vertical configuration and slightly linear following the BL without crossing the midline (Figs. 1, 3, 4).

Some of the lesions were covered with fine adherent scaling and Wickham`s striae (Figs. 1, 3).

Examination of the scalp, hair, oral mucosa and nails was within normal, without any apparent changes. 


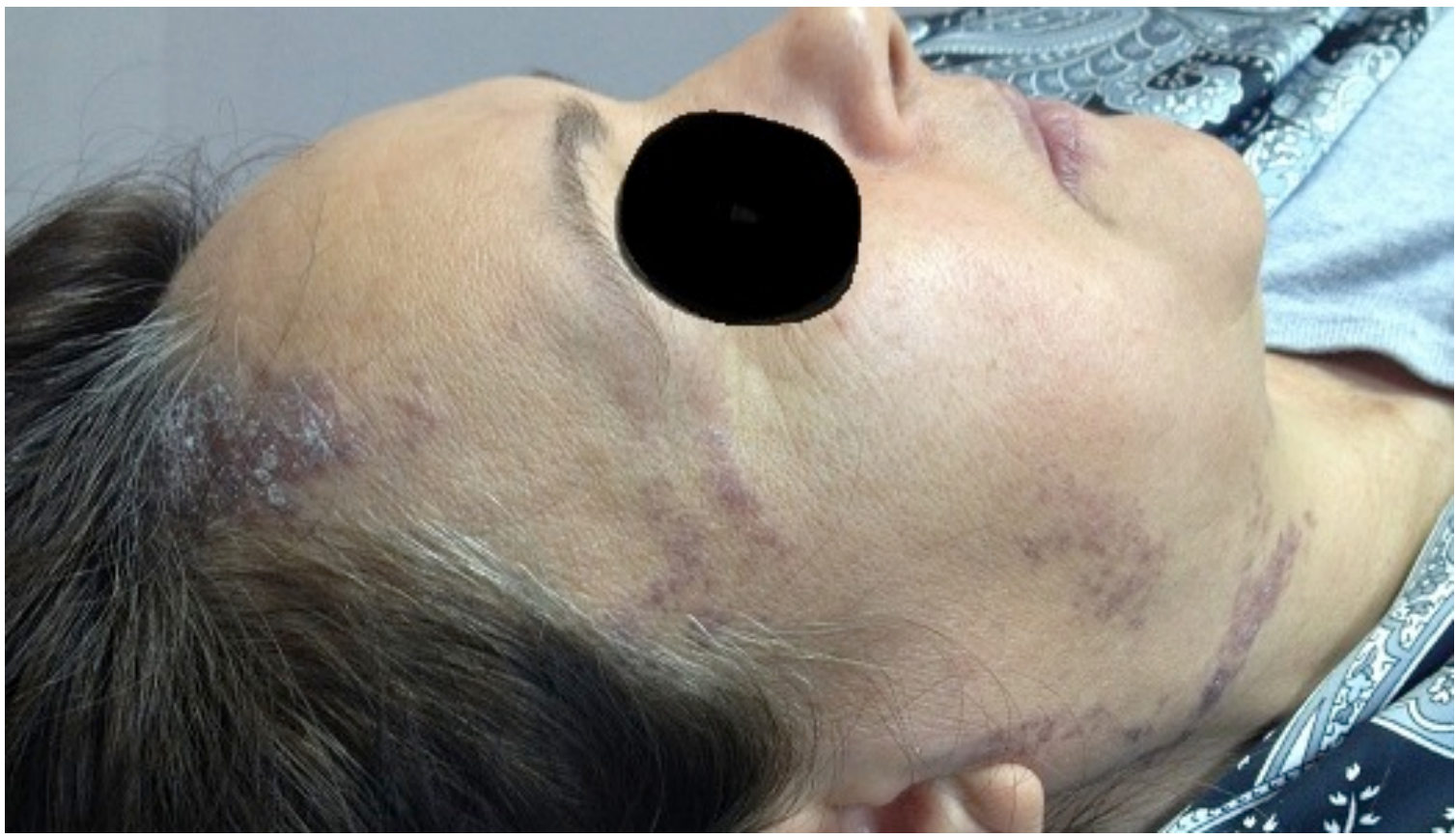

Fig. (1). Fine adherent scaling and Wickham`s striae.

Laboratory tests; namely complete blood count (CBC), with erythrocyte sedimentation rate (ESR), urea, electrolyte and creatinine $(\mathrm{U} / \mathrm{E} / \mathrm{C})$, liver function tests, thyroid function test, and urinalysis, were all within normal limits. Moreover, her viral screen including hepatitis $\mathrm{C}$ virus (HCV), hepatitis $B$ and Human immunodeficiency virus (HIV), was negative. Chest radiography was normal as no abnormality was found.

Her fasting blood sugar (FBS) was slightly raised (234 $\mathrm{mg} / \mathrm{dl}$ ), with slightly high glycoslated Hemoglobin (HbAlc) $8.8 \%$, and high cholesterol level 237 , triglyceride 123 , high density lipoprotein (HDL) 80 and low density lipoprotein (LDL) 132.4.

A differential diagnosis of lichen striatus, lichenoid herpes zoster, linear epidermal nevus and linear psoriasis, linear porokeratosis, linear Dariers disease, and inflammatory linear verrucous epidermal nevus (ILVEN) were considered. The histological readings differentiate between these differentials.

After ruling out some routine blood investigations, which were within normal limits, including the viral serology screen, a skin punch biopsy was taken and sent for histopathological (HP) examination.

A skin biopsy taken from a typical papule on the forehead and after staining with H\&E stain, it showed mild hyperkeratosis and hypergranulosis (Fig. 6a, b). Civatte bodies $^{1}$ were present in the epidermis (Figs. 6,7). There was also a dense band like lymphocytic infiltrate at the interface, with significant melanin incontinence (Fig. 7). Vacuolar degeneration of the basal layer is seen, as well as lymphocytic exocytosis (Fig. 6a, b). All of the readings were consistent with lichen planus. Immunofloresence was not performed due to the lack of availability of this service.

${ }^{1}$ Civatte bodies: are eosinophilic hyaline spheric bodies, which yielded due to apoptosis of individual basal cells.
Considering the clinical presentation and histopathologic findings, a final work up diagnosis of 'Blaschkoid LP' was made.

The patient was treated with medium potency steriods namely 'Betasone' ointment for one week. Lesions were gradually controlled and showed a response with post inflammatory brownish hyper pigmentation macules.

However, there was no response in the brownish pigmentation. She is still attending us for post inflammatory hyperpigmentation (PIH).

She came back for a follow up after 3 months for another skin problem, namely right plantar wart, and on examination of her earlier facial lesion, it had improved and flattened completely and some areas had completely recovered like the right forehead lesion (Fig. 2). However the rest, such as the one on the neck side and cheek remained pigmented (PIH).

\section{DISCUSSION}

Lichen planus is a common inflammatory dermatosis encountered in the dermatology setting and was first reported by Wilson in 1869; however linear unilateral lichen planus which follows Blaschko lines is a rare phenomenon [5]. It differs from the usual LP in absence of oral cavity involvement and the severe itching component $[2,4]$. Few cases have been reported in the existing literature, which mostly involved trunk side and to the best of my knowledge; this is the first reported case in an Amazaghi lady's head and neck, and the distinctive feature, it had involved one side of her face; the right side. Blashkoid LP is rare and represents less than $0.5 \%$ of LP $[3,6,8]$.

Amazighen; indicates a Berber root; an ethnic group indigenous to North Africa west of the Nile Valley, who they have their own languages and customs. 


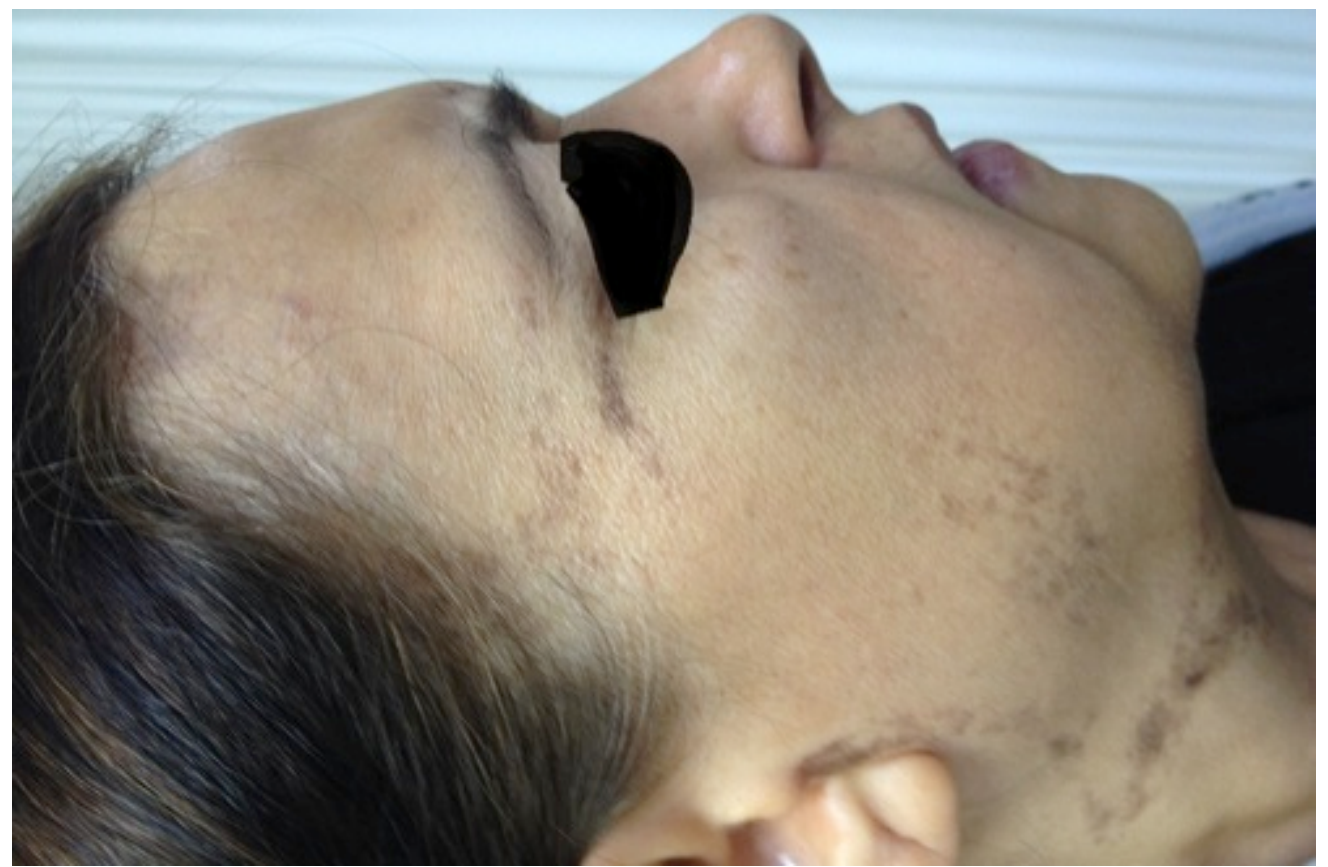

Fig. (2). Note the clearing of the lesion and PIH. The patient was treated with medium potency steroids cream and complete resolution noted after a week with residual post-inflammatory hyperpigmentation.

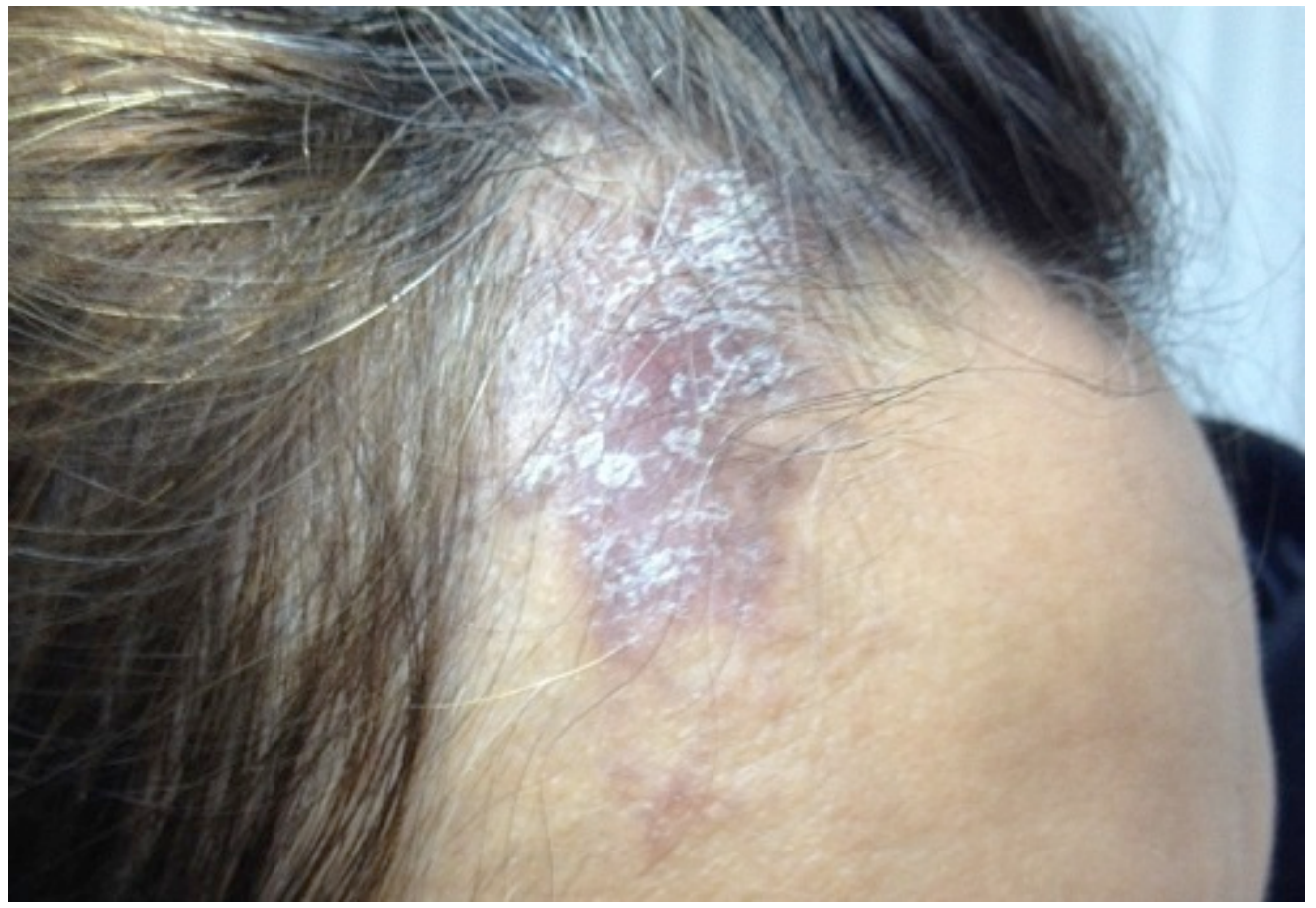

Fig. (3). Close-up views of the skin lesions. Physical examination showed multiple slightly hyperpigmented violaceous, discrete, raised papules and plaques in linear fashion. Notice the lesion scales on one side of the right forehead.

The literature did not examine if such cases have a predilection towards certain ethnicity, and reporting an Amazigh case might call for further research and investigation.

The skin lesion followed the dermatomal V1, V2, V3, C1 and $\mathrm{C} 2$, which coincides with the right apical part of the forehead near the hair line (1-3 mm), right temple, under right ear, and right cheek and the right neck side (Fig. 5).
The clinical and histological readings were suggestive of LP. Topical steroids (Betasone ointment) led to a remarkable improvement with PIH.

Many skin diseases whether congenital or acquired follow the pattern of Blaschko lines, such as vitiligo, incontinentia pigmenti and naevi, hypomelanosis of Ito and scleroderma [3, 4 and 6$]$.

Blaschko's lines, or Lines of Blaschko, are skin lines undetectable under normal conditions. They become 


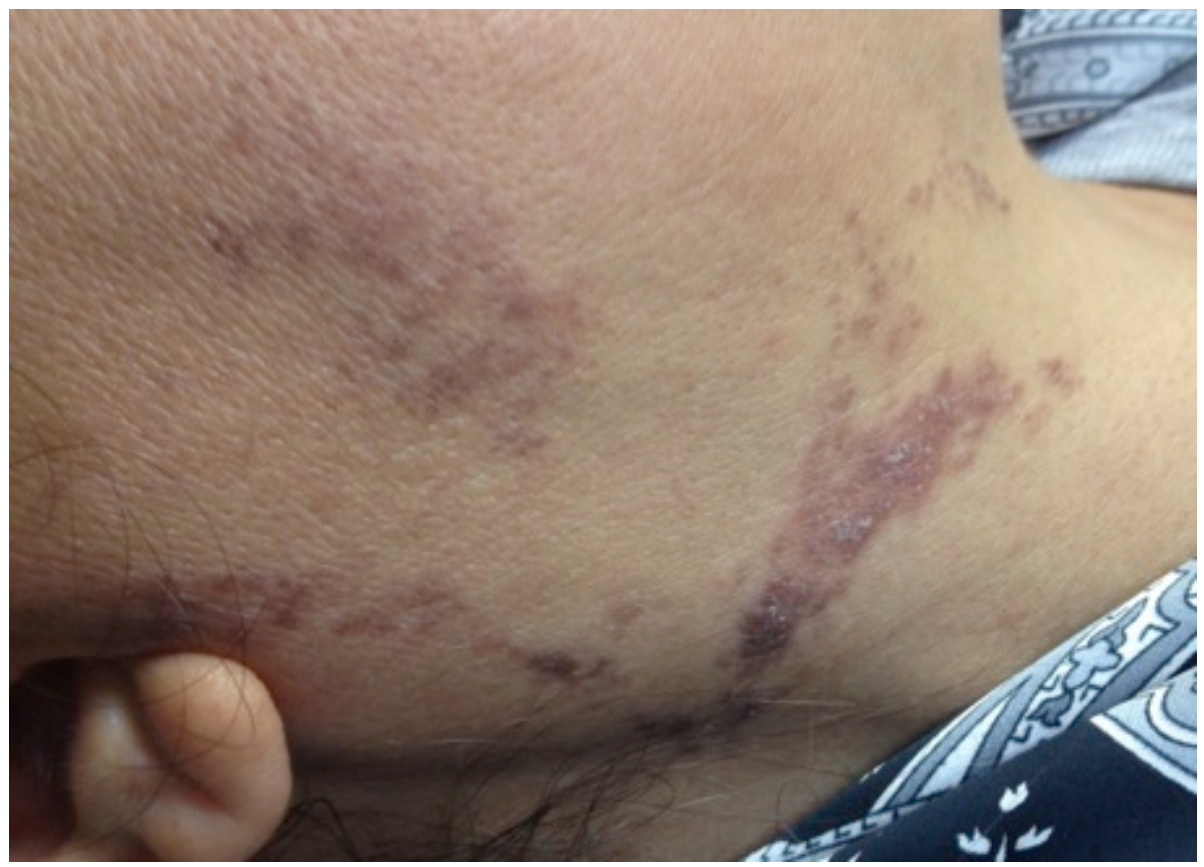

Fig. (4). Close up views; notice the linear lesion characteristics not crossing the mid line.

perceptible with some diseases of the skin or mucosa. They become apparent according to these line patterns. For instance; they pursue a "V" shape over the back, "S" shaped twists and spirals over the chest, stomach, and sides and curvy figures on the head. BL are distinguished from other linear patterns such as Voights, Langers and Lines of innervations of spinal nerves $[7,8]$.

Lichen planus (LP) is an acquired inflammatory disorder that can come with diverse faces [6]. Some have reported that LP followed a history of previous $\mathrm{HZ}$, and followed its pattern; however this case did not have such concomitant coincidence $[5,7]$.

In extremely rare cases, de novo linear skin lesions can occur on normal skin that has not been traumatized, as in this case $[4,7,8]$. The patient denied any history of $\mathrm{HZ}$ or trauma to her skin [7].
The cause of Blashkoid LP is indefinite; however it is assumed to be an abnormal keratinocyte clone which is unmasked after kicking off an episode for LP [8, 9].

Some studies have stated such a skin lesion might have a neural origin as it exhibits a dermatomal manner of involvement, but that suggestion was later on attributed to Blaschko lines (BL), which are considered to represent division of autonomic motor-visceral afferents or stretching of the skin during the embryogenesis period, and its distinctiveness does not cross the mid line of the body [5, 8]. Plus they do not correspond to the nervous system or vascular pathways [8]. Also it has been suggested there is a variety of human mosaicism where certain cell types react in different manners due to chromosomal aberrations [6, 8]. Moreover some have suggested those lines to be genetically programmed clones of aberrant cells during embryogenesis
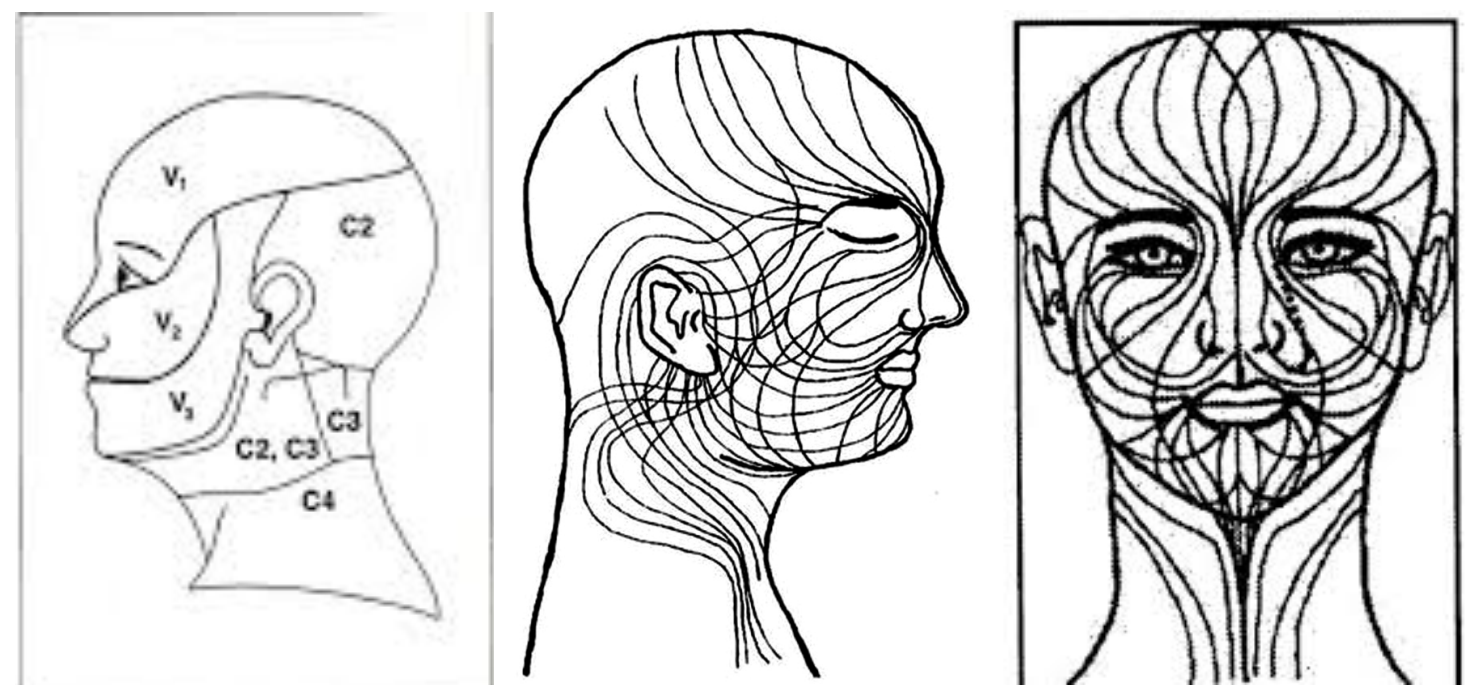

Fig. (5). Dermatome distribution and BLs, source: www.e-ijd.org. 
[6]. Others ascertained it is a form of acquired inflammation to BL (Blaschkitis) [3].

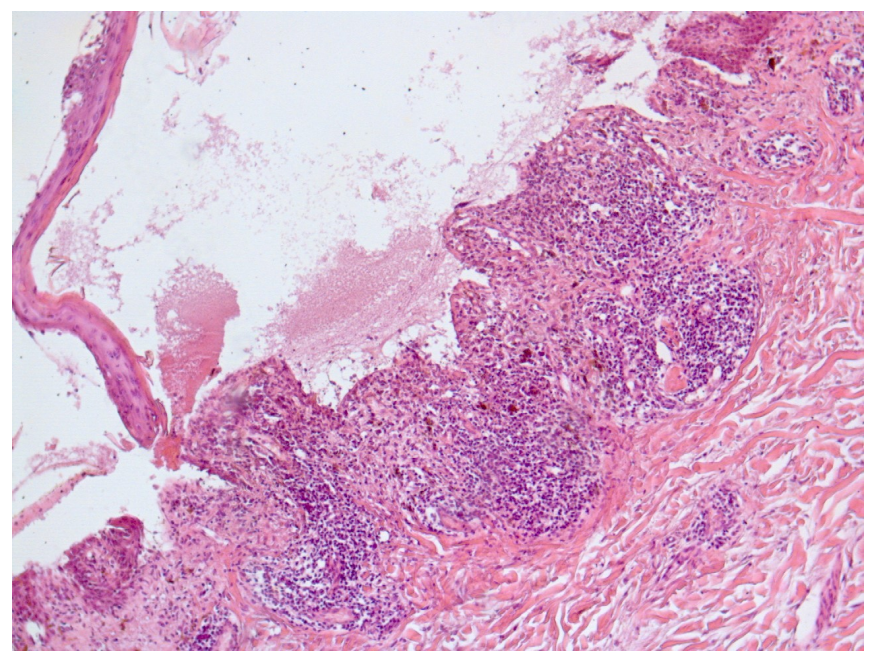

Fig. (6a). Note the band like infiltration in dermoepidermal junction and pinkish civatte bodies.

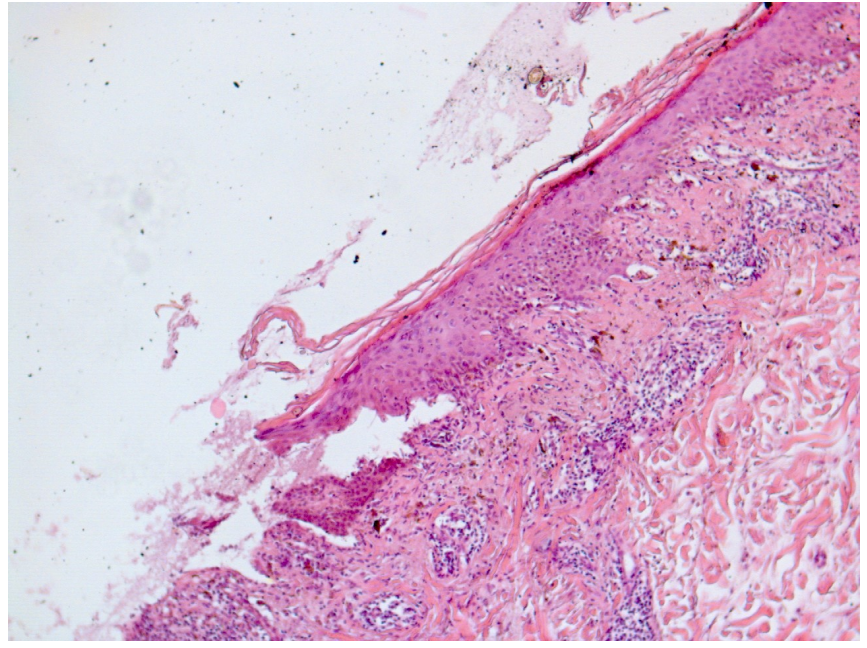

Fig. (6b). Note the Hyperkeratosis, and hypergranulosis. Also note the vacuolar changes.

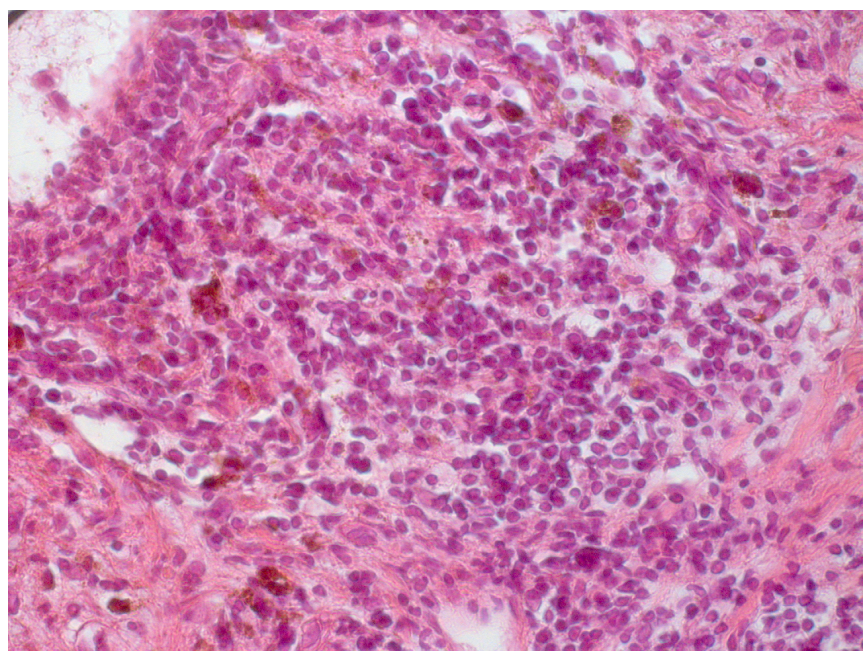

Fig. (7). Note the significant pigment incontinence (H\&E stain $\mathrm{x} 40$ ).
The confusing part is this arrangement of the lesion was along Blaschko's lines and could be misinterpreted as that of zosteriform skin lesions [9-11].

'BL' the concept was first explained by Alfred Blaschko (German dermatologist) in 1901 at the 7th Congress of German Dermatological Society [3, 4, 8].

Her past medical history was significant for diabetes mellitus, and this could be the only co-morbidity, as her glycoslated Hemoglobin (HbAlc) was slightly high at $8.8 \%$, which indicate uncontrolled diabetes in the last three months. She does not recall any triggering factor such as shingles. Family history was noncontributory.

To conclude, Blaschkoid LP is usually benign and selflimiting, and the absence of upsetting itchiness element in many patients of this LP variant, would delay patient seeking medical advice and thus delay diagnosis, and this could be attributing to the actual missing report of such cases in the existing literature.

Dermatologists must be aware of this condition, so the diagnosis can be made at an early stage, improving the patient's quality of life.

\section{LIST OF ABBREVIATIONS}

$\begin{array}{ll}\mathrm{BL} & =\text { Blaschko line } \\ \mathrm{CBC} & =\text { Complete blood count } \\ \mathrm{CMI} & =\text { The cell-mediated immunity } \\ \mathrm{ESR} & =\text { Erythrocyte sedimentation rate } \\ \mathrm{FBS} & =\text { Fasting blood sugar } \\ \mathrm{HbA1c} & =\text { Glycated hemoglobin } \\ \mathrm{HCV} & =\text { Hepatitis C virus } \\ \mathrm{HDL} & =\text { High density lipoprotein } \\ \mathrm{HIV} & =\text { Human immunodeficiency virus } \\ \mathrm{HP} & =\text { Histopathological } \\ \mathrm{HZ} & =\text { Herpes zoster } \\ \mathrm{ILVEN} & =\text { Inflammatory linear verrucous epidermal } \\ \mathrm{LDL} & =\text { nevus } \\ \mathrm{LP} & =\text { Lichen planus } \\ \mathrm{PIH} & =\text { Post inflammatory hyperpigmentation } \\ \mathrm{U} / \mathrm{E} / \mathrm{C} & =\text { Urea electrolyte and creatinine }\end{array}$

\section{CONFLICT OF INTEREST}

The author confirms that this article content has no conflict of interest.

\section{ACKNOWLEDGEMENTS}

A special acknowledgment goes to my mentor in my Diploma in the UK, Dr Olive frost for her proof reading and revising it critically for important intellectual content.

\section{REFERENCES}

[1] Sharma R, Maheshwari V, Chandra M. Unilateral multiple linear lichen planus along the lines of blaschko: A report of two cases. Indian J Dermatol Venereol Leprol 1999; 65(5): 225-6. 
[2] Kavoussi H, Ramazani M, Salimi E. Lichen planus along with Blaschko lines, "Blaschkoian lichen planus". J Pak Assoc Dermatol 2013; 23 (2): 153-8.

[3] Jackson R. The lines of Blaschko: a review and reconsideration: Observations of the cause of certain unusual linear conditions of the skin. Br J Dermatol 1976; 95 (4): 349-60.

[4] Miljkovi J, Beli M, Godi A, Klemenc P and Marin J. Zosteriform lichen planus-like eruption. Acta Dermatoven APA 2006; 15 (2) 94-7.

[5] Gupta AK, Gorsulowsky DC. Unilateral lichen planus: an unusual presentation. Arch Dermatol 1987; 123: 295-6.

[6] Gupta S, Gupta S, Thomas M, Mahendra A. Unilateral lichen planus along the lines of Blaschko: a rare clinical presentation. Acta Medica Indonesiana 2012; 44 (2): 163-4.
[7] Lade NR, Saoji V, Singh AL. Blaschkoid lichen planus: not a Koebner phenomenon. Dermatol Online J 2013; 19 (4): 17.

[8] Rather PA, Hassan I. blaschkoid lichen planus in an adult kashmiri male: a rare presentation. Our Dermatol Online 2013; 4 (2): 17982.

[9] Perry D, Fazel N. Zosteriform lichen planus. Dermatol Online J 2006; 12: (5): 3 .

[10] Ber Rahman S, Ul Bari A, Mumtaz N. Unilateral blaschkoid lichen planus involving the entire half of the body, a unique presentation. Dermatol Online J 2007; 13(3): 36.

[11] Arfan-ul-B, Rahman SB. Zosteriform lichen planus. J Coll Phys Surg Pak 2003; 13 (2): 104-5.

(C) Ebtisam Elghblawi; Licensee Bentham Open.

This is an open access article licensed under the terms of the Creative Commons Attribution Non-Commercial License (http://creativecommons.org/licenses/by-nc/3.0/) which permits unrestricted, non-commercial use, distribution and reproduction in any medium, provided the work is properly cited. 